\title{
miRNAs in the Pathogenesis of Systemic Lupus Erythematosus
}

\section{Bo Qu ${ }^{1}$ and Nan Shen ${ }^{1,2,3, *}$}

1 Department of Rheumatology, Renji Hospital, School of Medicine, Shanghai Jiao Tong University, Shanghai 200001, China; E-Mail: qubo.bio@gmail.com

2 Institute of Health Sciences, Shanghai Institutes for Biological Sciences (SIBS) \& Shanghai Jiao Tong University School of Medicine (SJTUSM), Chinese Academy of Sciences (CAS), Shanghai 200031, China

3 Division of Rheumatology and the Center for Autoimmune Genomics and Etiology (CAGE), Cincinnati Children's Hospital Medical Center, Cincinnati, OH 45229-3039, USA

* Author to whom correspondence should be addressed; E-Mail: Nan.Shen@cchmc.org; Tel.: +86-21-6326-0477; Fax: +86-21-6373-0455.

Academic Editors: Chak-Sing Lau and Vera Sau-Fong Chan

Received: 24 March 2015 / Accepted: 20 April 2015 / Published: 28 April 2015

\begin{abstract}
MicroRNAs (miRNAs) were first discovered as regulatory RNAs that controlled the timing of the larval development of Caenorhabditis elegans. Since then, nearly 30,000 mature miRNA products have been found in many species, including plants, warms, flies and mammals. Currently, miRNAs are well established as endogenous small ( $22 \mathrm{nt})$ noncoding RNAs, which have functions in regulating mRNA stability and translation. Owing to intensive investigations during the last decade, miRNAs were found to play essential roles in regulating many physiological and pathological processes. Systemic lupus erythematosus (SLE) is a chronic autoimmune disease characterized by elevated autoantibodies against nuclear antigens and excessive inflammatory responses affecting multiple organs. Although efforts were taken and theories were produced to elucidate the pathogenesis of SLE, we still lack sufficient knowledge about the disease for developing effective therapies for lupus patients. Recent advances indicate that miRNAs are involved in the development of SLE, which gives us new insights into the pathogenesis of SLE and might lead to the finding of new therapeutic targets. Here, we will review recent discoveries about how miRNAs are involved in the pathogenesis of SLE and how it can promote the development of new therapy.
\end{abstract}


Keywords: microRNA; systemic lupus erythematosus; innate immunity; adaptive immunity; lupus nephritis; biomarker; therapy

\section{The Biology of miRNA}

miRNA genes are mainly located within intronic regions of a genome, with a few in exonic regions [1]. miRNAs tend to be encoded in a clustered manner, such that nearly 50\% of mammalian miRNA loci are found adjacent to other miRNAs [2]. Clustered miRNAs can either be transcribed as one primary transcript and regulated by a common promoter or transcribed individually using different promoters [3]. Although a few miRNAs associated with Alu repeats can be transcribed by RNA polymerase III (Pol III) [4], Pol II is used to transcribe most miRNA genes [5]. After being transcribed, primary transcripts of an miRNA gene (pri-miRNAs) are subjected to cleavage by Drosha in the nucleus [6], which is the first step of the miRNA maturation process. Subsequently, a small hairpin RNA (pre-miRNA) is released and exported to the cytoplasm by Exportin-5 [7]. In the cytoplasm, pre-miRNAs are the substrates of another RNase III, Dicer. With the help of other double-stranded RNA-binding domain (dsRBD) proteins, Dicer dices the pre-miRNA into a $\sim 22$ nt-long duplex RNA, which contains two mature miRNA strands [8]. Based on their thermodynamic properties, one of the two strands will be preferred to incorporate into the RNA-induced silencing complex (RISC) that contains the Argonaute (Ago) family protein that is responsible for exerting the inhibitory function of the miRNA [9]. The other strand, which is called miRNA* and exists at a very low level, was considered to have no function and finally to be degraded by the cell. However, more and more evidence indicates that these miRNA* strands can indeed accumulate to a certain level and are functional in some cases [10,11]. (Therefore, the use of the $\mathrm{miR} / \mathrm{miR}^{*}$ nomenclature is ceased, and instead, $-5 \mathrm{p}$ or $-3 \mathrm{p}$ was used for the sequences derived from the $5^{\prime}$ or $3^{\prime}$ arms of the hairpin precursor [12]. However, we will still use the old nomenclature in order to be consistent with the original research paper).

miRNAs are mainly regulated at the transcription level, which gives them a cell type-specific and spatiotemporal expression pattern [13]. Many characteristics of miRNA gene promoters are similar to those of protein coding genes, and thus, miRNAs can be regulated by the mechanisms that are found to take part in regulating protein coding genes, such as the binding of transcription factors or enhancers to the cis regulatory elements, DNA methylation or histone modification status of the promoter [14]. Moreover, the expression of miRNAs can also be regulated post-transcriptionally. In the nucleus, Drosha needs $D G C R 8$ (DiGeorge critical region 8) to facilitate pri-miRNA processing [15]; while several other accessory factors, such as EWSR1, DDX5 and DDX17, can promote the fidelity and activity of Drosha processing [16]. In the cytoplasm, like Drosha, Dicer needs other dsRBD proteins, TRBP, to dice pre-miRNAs into the miRNA duplex [17]. Other proteins can also affect miRNA maturation by regulating these accessory components in miRNA processing. For example, TRIM71 was found to regulate let-7 biogenesis by ubiquitinating $\operatorname{Lin} 28 \mathrm{~B}$, which regulated let-7 maturation by recruiting TUT4 to block Dicer function $[18,19]$.

By base-pairing of its seed region (nucleotides 2-8 from the 5' end of a miRNA) to the 3'UTR of a target mRNA or to the coding regions of a target mRNA, mature miRNAs mediate the binding of 
RISC to their targets, then inhibit the translation of the mRNAs and even cause degradation of the mRNAs [20]. Each mature miRNA has the ability to target multiple different mRNAs. On the contrary, a particular mRNA can be targeted by multiple miRNAs that regulate the expression of the same gene in concert.

Similar to miRNA biogenesis, many factors can affect miRNA's binding to its target mRNA and its subsequent inhibitory function. As for miRNA itself, nucleotides in the middle of an miRNA could have influence on its targeting relationship with mRNAs [21]. RNA-binding proteins (RBPs), such as DND1 and HuR, which bind to the 3'UTR of a mRNA, can interfere with RISC's binding to its target and protect certain mRNAs from miRNA-mediated repression [22,23]. There is evidence that circular RNAs (circRNAs) serve as potential regulatory factors for both the level and function of miRNAs [24]. Ago proteins in RISC are one of the regulatory targets, which are used to control miRNA function. TRIM71, a member of the TRIM-NHL family proteins, can directly associate and ubiquitinate Ago2 protein and, thus, regulate its turnover [25].

By fine-tuning gene expression, miRNAs can function as key regulatory factors in many biological processes, including cell proliferation, cell differentiation, apoptosis, organogenesis, development and immune responses [26,27]. Abnormal expression or function of miRNAs has been found in many diseases, such as cancer, heart diseases and autoimmune diseases [28-30].

\section{2. miRNAs in the Pathogenesis of SLE}

It is interesting that autoimmune responses to some key components of the miRNA pathway were found in systemic lupus erythematosus (SLE), rheumatoid arthritis (RA) and Sjögren's syndrome [30]. In SLE, it was reported that anti-Su autoantibodies, which were usually found in lupus patients, could recognize the catalytic enzyme in miRNA pathways (Ago1, Ago2, Ago3, Ago4 and Dicer) [31,32], which indicated the possible mutual relationship between miRNA and the pathogenesis of SLE. miRNA expression profiles using samples from a lupus mouse model and lupus patients revealed the clinical relevance of miRNAs in SLE [33,34]. Investigation of the function of those abnormally-expressed miRNAs sheds light on the mechanism of the development of SLE.

\section{3. miRNAs Regulate Innate Immunity in SLE}

Innate immune cells do not produce antigen-specific antibodies or receptors. However, by utilizing pattern recognition receptors (PRRs), innate immune cells can recognize dangerous signals derived from pathogens or damaged cells [35]. There are several PRRs families in the innate immune system: toll-like receptors (TLRs), NOD-like receptors (NLRs), RIG-I-Like receptors (RLRs), cytosolic DNA receptors (CDS); and ligand binding of these receptors triggers signaling cascades, which activate downstream transcriptional factors (such as NFKB and/or IRFs) and lead to the production of inflammatory cytokines and/or type I interferons [36-38]. These pro-inflammatory cytokines will further act in autocrine or paracrine manners to mediate the inflammation of local tissue, the recruitment and activation of many other immune cells and the initiation of the adaptive immune system [39].

The involvement of innate immunity in SLE pathogenesis has long been established [40]. The evidence that activation of innate receptor TLR 9 co-stimulated auto-reactive B-cells provided an explanation to epitope spreading in SLE [41]. Subsequent researches demonstrated that activation of 
$T L R 9$ accelerated renal disease in a lupus mouse model, MRL/MpJ-Fas ${ }^{\mathrm{lpr}} / \mathrm{J}$ mice (common name MRL-lpr, mouse model that has spontaneous mutation of FAS gene and is prone to SLE) [42]. Type I interferon is recognized as one of the key pro-inflammatory cytokines in the pathogenesis of SLE [43]. Due to their key roles in stimulating downstream type I interferon production, TLRs, RLRs and CDSs on innate immune cells are recognized as important factors that participate in the initiation and augmentation of autoimmune responses in SLE [44,45]. TLR7 was found to be essential for producing type I interferon and the development of lupus symptoms in an induced SLE model [46]. Consistently, translocation of $T L R 7$ to the Yaa locus accelerated systemic autoimmunity in another murine lupus model [47]. Many cytosolic DNA sensors could be induced by interferon and were found elevated in lupus patients [48]. Chronic activation of the STING-dependent cytosolic DNA sensing pathway may also be responsible for type I interferon production in SLE [49]. Several studies discovered that mutations in human TREXI were related to SLE [50]. TREX1-deficient mice showed elevated production of type I interferon and lupus-like symptoms, which was demonstrated to be mediated by STING-dependent pathways [51,52]. Tight regulation of the innate immune signaling pathway keeps our immune responses in control, protecting us from autoimmune diseases.

Several miRNAs were found to have crucial roles in negative regulating innate immune responses. Transcription of miR-146a could be induced by the engagement of several TLRs and pro-inflammatory cytokines, which stimulated downstream NFkB activity [53]. By targeting signaling adaptor proteins the TNF receptor-associated family (TRAF)-6 and IL-1 receptor-associated kinase (IRAK)-1, miR-146a suppresses NFאB activation and subsequent cytokine production [53]. miR-146a could also be able to inhibit type I interferon induction by TLR7 and RIG-I pathway [54,55]. Thus, $m i R-146 a$ can suppress type I interferon production by targeting multiple key molecules in the innate signaling pathway. Profiling of the miRNAs expressed in the PBMCs from lupus patients revealed that miR-146a was under-expressed in SLE [55]. Additionally, this was possibly due to a germline genetic variant in the $m i R-146 a$ promoter [56]. Further investigation found that STAT1 was another target of miR-146a, and there was a reverse correlation of $m i R-146 a$ levels with the expression of interferon-inducible genes and SLE disease activity, indicating a critical role of $m i R-146 a$ in excessive type I interferon production and signaling activity in SLE [55].

miR-155 was another miRNA that has been investigated intensively for its function in regulating innate immune response. By targeting MYD88 and TAB2, miR-155 could inhibit inflammatory response [57,58]. On the contrary, $m i R-155$ promoted inflammatory response and type I interferon signaling by targeting the suppressor of cytokine signaling-1 (SOCS-1) in macrophage [59], which indicated the context-dependent character of the regulatory function of miR-155. One interesting discovery is that although originating from the same precursor, $m i R-155^{*}$ has an opposite effect on the regulation of type I interferon production compared to that of $m i R-155$ in pDCs (plasmacytoid dendritic cells) [60]. When ligands bind to TLR7 on pDCs, the transcription of the $m i R-155 / m i R-155^{*}$ gene is activated, and this leads to rapid production of mature $m i R-155^{*}$, which increases the ratio of $m i R-155^{*}$ to $m i R-155$. Thus, $m i R-155^{*}$ facilitates type I interferon production by inhibiting IRAKM expression. Type I interferon produced downstream of $T L R 7$ acts in an autocrine/paracrine manner to induce and activate KSRP, which will promote $m i R-155$ maturation, but not $m i R-155^{*}$. As $m i R-155$ accumulates, which inhibits TAB2 in pDCs, the production of type I interferon and $\mathrm{pDC}$ activation are limited to a proper level. pDCs have been documented as the cells with great the ability to produce type I IFN 
when encountering nucleic acid, which could be recognized by the TLRs of pDCs. pDCs have been associated with SLE and might contribute to its pathogenesis by recognizing self-DNA or RNA, then producing a huge amount of pathogenic type I IFN [61]. Recent studies demonstrated that depletion of $\mathrm{pDCs}$ in BXSB/MpJ lupus-prone mice (males of this mouse model has mutant Yaa containing $\mathrm{Y}$ chromosome and develops severe form of SLE) ameliorated lupus pathology, which indicated that pDCs played critical roles during the IFN-dependent initiation of lupus and might be an attractive therapeutic target for treating SLE [62]. Considering the key role of type I interferon in SLE pathogenesis and pDCs' strong capability of secreting type I interferon, further investigation of the expression of $m i R-155$ and $m i R-155^{*}$ in pDCs in SLE will shed light on the mechanism of the deregulated type I interferon production.

Other miRNAs also play a part in regulating innate immune responses. miR-3148 could target TLR7 through binding to its 3 'UTR, which provides us with an explanation of how a genetic variation found in the 3'UTR of TLR7 mRNA affects its expression in SLE [63]. DCs from Blimp1-deficient mice showed increased expression of let-7c, which is responsible for the pro-inflammatory phenotype in DCs, and a similar regulatory mechanism has been found in human [64]. In addition, let-7a, the expression of which is elevated in the kidneys of SLE patients and in the mesangial cells of lupus mice, could promote E2F-mediated cell proliferation and the activation of NFאB [65].

\section{4. miRNAs Regulate Adaptive Immunity in SLE}

Due to the fact that autoantibodies are elevated in SLE, auto-reactive B-cells and T-cells are recognized to be important in disease pathogenesis and have been studied intensively and extensively [66,67]. Aberrant B-cell tolerance happens at multiple levels, which combines with deregulated BCR and BAFF signaling to promote the activation of auto-reactive B-cells $[68,69]$. Due to their ability to regulate B-cell responses, CD4+ T-cells were also found to have defects and to contribute to the abnormal adaptive immunity in SLE. CD4+ T-cells from SLE patients were demonstrated to have aberrant DNA hypomethylation, which provided an epigenetic explanation for excessive T-cell activation, but the mechanism was still unclear [70]. The miRNA profile of CD4+ T-cells from MRL-lpr mice identified $m i R-21$ and $m i R-148 a$ as two upregulated miRNAs [71]. Further investigation found that $m i R-21$ and $m i R-148 a$ could considerably reduce the DNMT1 protein level, which was one of the major epigenetic components that had been linked to DNA hypomethylation in T-cells in SLE [72]. Additionally, $m i R-21$ inhibited RAS-MAPK-ERK signaling upstream of DNMT1 in T-cells. Considering that $m i R-21$ was found to be upregulated in PBMCs in SLE patients and correlated with disease activity, it is possible that elevated $m i R-21$ and $m i R-148 a$ contribute to the hypomethylation status of T-cells in SLE. Besides $m i R-21$ and $m i R-148 a, m i R-126$ was also reported to modulate DNA methylation in SLE CD4+ T-cells by directly targeting DNMT1 [73], which provided us a more comprehensive explanation of DNA methylation in CD4+ T-cells in SLE. miR-29b, which is upregulated in SLE CD4 ${ }^{+}$T-cells, has been shown to inhibit SP1 expression in human T-cells, meaning that they inhibit the expression of $D N M T 1$ and modulate DNA methylation [74], and further studies demonstrated that inhibition of miR-29b in the T-cells of SLE patients reversed DNA hypomethylation and the upregulation of downstream genes.

Inflammatory cytokines and chemokines are dysregulated and have crucial roles in the development of SLE. For example, the expression of IL2 in T-cells from SLE patients is dramatically lower than that 
from healthy controls [75]. IL2, mainly functioning in a paracrine manner, was proposed to have an indispensable role of maintaining natural regulatory T-cells in the periphery [76] and, thus, could contribute to the uncontrolled immune responses. CCL5 (or RANTES) is one of the many overexpressed chemokines in sera from lupus patients [77]. CCL5 plays a critical role in regulating immune cell movement and is indicated to have functions in lupus-associated tissue damage [78]. In addition to the transcription regulators, such as CREM (cAMP-responsive element modulator), CREB1 (CREM-binding protein 1) and NFAT (NFkB, AP1 and nuclear factor of activated T-cells), it was shown that miR-31, downregulated in SLE T-cells, could repress the expression of RhoA, a negative regulator of NFAT, and was responsible for impaired IL2 expression [79,80]. Moreover, the expression of $m i R-31$ was inversely correlated with RhoA in SLE patients [80]. Another miRNA, miR-125a, was found significantly downregulated in PBMCs from SLE patients [81]. Because of its selective expression in T-cells and its target relationship with KLF13, miR-125a was suggested to promote the secretion of CCL5 by SLE T-cells [81]. Thus, other than epigenetic traits, miRNAs were also involved in regulating cytokine production in T-cells in SLE.

The autoantibodies in lupus patients are high-affinity, somatically-mutated and Ig-switched, indicating that there were defects of germinal center (GC) responses in SLE [82]. Tfh cells (follicular helper T cells), derived from the differentiation of naive T-cells, mediate memory and plasma B-cells formation in GC (germinal center), and crosstalk between Tfh cells and B-cells will lead to class switching and affinity maturation of B-cells [83]. So far, studies of miRNAs in Tfh cell differentiation have been mainly focused on the miR-17 92 cluster. Mice that have the transgene of the miR-17 92 cluster developed lymphoproliferative disease and autoimmune symptoms, like SLE [84]. On the molecular level, miR-17 92 was shown to repress the expression of PTEN and PHLPP2 and regulate Tfh cell differentiation and function $[85,86]$. As for B-cells, researchers found that elevated expression of $m i R-30 a$ was responsible for the underexpression of LYN in SLE B-cells [87]. Additionally, more efforts are need to investigate miRNAs in B-cells.

\section{5. miRNAs Regulate Inflammation in Tissue-Resident Cells in SLE}

Usually, SLE patients have multiple clinical features and organ damage caused by inflammation. IL17 is one of the most studied pathogenic pro-inflammatory cytokines, and it has been shown to be overexpressed in target tissues and to be responsible for local inflammation in many autoimmune diseases, such as RA, multiple sclerosis and SLE [88-90]. miR-23b was found underexpressed in target tissues in a study that comprehensively profiled miRNA expression in affected tissues of patients with SLE or RA, as well as mouse models of SLE or RA [91]. Subsequent results showed that $m i R-23 b$ could target $T A B 2, T A B 3$ and IKK- $\alpha$ to inhibit IL17, TNF or IL1 $\beta$ signaling. These findings emphasized the regulatory role of miRNAs in tissue-resident cells. Lupus nephritis is one of the major complications associated with SLE [92]. Besides immune disorders, renal tissue also plays an important role in local inflammation. miRNA expression profiles of renal tissue, which identified 36 upregulated and 30 downregulated miRNAs in the renal tissues of lupus nephritis patients, provided a good source of differentially-expressed miRNAs in lupus kidney tissues [93]. Recent results showed that let-7a was significantly increased in mesangial cells of NZBWF1/J mice (common name NZB/W, this mouse model develops an autoimmune disease resembling human SLE) compared to age-matched NZW/LacJ 
mice (common name NZW, F1 hybrids of NZW/LacJ and NZB/BINJ are used as a model for human SLE) and might be involved in regulating IL6 production in these cells [65]. Other than regulating inflammation, miRNAs were found to mediate renal fibrosis in lupus nephritis. By comparing miRNA expression in kidney biopsies of lupus nephritis patients, researchers identified miR-150 with a positive correlation with chronicity scores and the expression of profibrotic proteins [94]. Furthermore, they found that TGF $\beta 1$ could induce miR-150, and elevated miR-150 could target antifibrotic protein SOCS1 with upregulated profibrotic proteins in renal proximal tubular and mesangial cells. These findings suggested that TGF $\beta 1$ executed its profibrotic effects partly through $m i R-150$.

\section{6. miRNAs as Biomarkers of SLE}

Over the past few decades, tremendous efforts have been devoted to tackling the mechanisms for the initiation and development of the autoimmunity and subsequent multiple organ damage of SLE. Although significant progress has been made, there are still many other challenges to conquer the disease. One of those important needs is the lack of reliable biomarkers for diagnosis, monitoring, stratification and prediction of prognosis [95].

As the investigation of the functions of lupus-associated miRNAs goes on, it has been recognized that miRNAs are dysregulated in SLE [33,96,97]. Recent studies showed that there were distinct expression patterns of miRNAs in peripheral blood leukocytes of SLE patients. Additionally, these specific patterns were found to be associated with different autoantibody compositions in those SLE patients [98]. Another study identified aberrant expression of miRNAs (especially hsa-miR-371-5p, hsa-miR-423-5p, hsa-miR-638, hsa-miR-1224-3p and hsa-miR-663) in the PBMCs of lupus nephritis patients across different patients with different races [99]. With the discovery of the existence of miRNAs in body fluid, miRNAs are becoming an ideal biomarker for many diseases [100,101]. Serum and urinary miRNAs were shown to be associated with different disease characteristics of SLE [102]. Another study demonstrated that circulating miRNAs were found systematically altered in SLE, and a 4-miRNA signature could be used to discriminate SLE with healthy controls, with another set of miRNA associated with lupus nephritis [103]. With the development of the detection technique for circulating miRNA, new technologies used for profiling miRNA expression and more expression data obtained, miRNA will possibly become a convenient biomarker choice for SLE and its associated organ damage [97,104].

\section{The Therapeutic Perspective of miRNAs in SLE}

Besides the basic research on the mechanisms for the pathogenesis of SLE, there are many research works focused on how to translate the knowledge of miRNAs in SLE into the development of novel therapies. Recent studies revealed that the active metabolite of mycophenolate mofetil (MMF), which is widely used for the treatment of SLE, could upregulate the expression of $m i R-142-3 p / 5 p$ and $m i R-146 a$ in T-cells [105]. This finding suggested that the therapeutic effects of MMF might partially be mediated by these two miRNAs and that we could develop more specific therapeutic methods by using these two miRNAs. Since we have known the mechanisms of certain miRNAs regulating the abnormal activation of the immune responses in SLE, there have been many efforts made to explore the possibility of using miRNA as therapeutic targets or treatment reagents for SLE. miR-155 has long been established as 
a regulator of B-cell functions [106,107]. Based on these findings, miR-155 might take part in regulating the production of autoantibodies in SLE. In fact, ablation of miR-155 in MRL-lpr lupus-prone mice reduced autoantibody production with the alleviation of kidney inflammation [108]. Another research work demonstrated the deficiency of miR-155 in mice protected them from Pristane-induced lupus-associated pulmonary hemorrhage and application of a synthetic miR-155 antagomir in the mice could ameliorate pulmonary hemorrhage induced by Pristane [109]. Recent studies showed that overexpression of miR-155 could inhibit the expression of PP2Ac, which negatively regulates the expression of IL2 in PBMCs. Thus, the overexpression of $m i R-155$ could possible relieve the inhibition of IL2 by PP2Ac in juvenile SLE disease [110]. These results indicate that blocking miR-155 might benefit SLE patients. Further, in vivo silencing of $m i R-21$ by seed-targeting LNA altered the ratios of $\mathrm{CD} 4 / \mathrm{CD} 8 \mathrm{~T}$-cell-reversed splenomegaly and reduced the number of Fas receptor-expressing lymphocytes [111]. Another promising miRNA is $m i R-146 a$, which is recognized as a major negative regulator of immune response, and its deficiency led to multiple immune disorders [53,112,113]. Consistent with previous findings of $m i R-146 a$ 's regulatory functions in immune response, injection of miR-146a agomirs, a chemical-modified miR-146a mimic, rendered the mice resistant to Pristane-induced hemorrhagic pulmonary capillaritis with suppressed interferon response [114]. As an example of miRNA-based therapy, inhibitors of $m i R-122$ are used in clinical trials to test their activity in HCV (hepatitis C virus) infection [115]. Although there are still issues that need to be solved, such as developing efficient delivery approaches and chemical modifications for the improvement of the absorption and the stability of synthetic oligonucleotides, as well as testing the pharmacokinetic traits and side effects of administrating miRNA mimics or inhibitors in vivo, utilizing miRNAs as therapeutic molecules is still a promising choice to treat SLE patients in the future [115]. (Table 1 summarizes the targets and the signaling pathways of the miRNAs in SLE discussed above).

Table 1. miRNAs in the pathogenesis of systemic lupus erythematosus (SLE).

\begin{tabular}{cccc}
\hline miRNA & Target & Regulated Process & References \\
\hline let-7a & IL6 & IL6 induction & {$[65]$} \\
let-7c & Blimp1, SOCS1 & Activation of DCs & {$[64]$} \\
miR-125a & KLF13 & CCL5 induction in T-cells & {$[81]$} \\
miR-126 & DNMT1 & DNA methylation in T-cells & {$[73]$} \\
miR-146a & TRAF6, IRAK1 & NFkB mediated inflammatory response & {$[53]$} \\
& TRAF6, IRAK1, IRAK2 & RIG-I-dependent anti-viral pathway & {$[54]$} \\
& IRF5, STAT1 & Type I IFN induction and signaling & {$[55]$} \\
miR-148a & DNMT1 & DNA methylation in T-cells & {$[71]$} \\
miR-150 & SOCS1 & Renal fibrosis & {$[94]$} \\
miR-155 & MyD88, TAB2 & TLR/IL1 inflammatory pathway & {$[57,58]$} \\
& SOCS1 & Type I IFN signaling & {$[59]$} \\
miR-155* & PP2Ac & IL2 induction & {$[110]$} \\
miR-17 92 & IRAKM & Type I IFN induction & {$[60]$} \\
& PTEN, Bim & Proliferation of lymphocytes & {$[84]$} \\
miR-21 & Rora, PHLPP2 & Differentiation and function of Tfh cells & {$[85,86]$} \\
miR-23b & RASGRP1 & DNA methylation in T-cells & {$[71]$} \\
miR-29b & TAB2, TAB3, IKK $\alpha$ & IL17, TNF $\alpha$, IL1 $\beta$ signaling & {$[91]$} \\
\hline
\end{tabular}


Table 1. Cont.

\begin{tabular}{cccc}
\hline miRNA & Target & Regulated Process & References \\
\hline miR-30a & Lyn & Activation of B-cells & {$[87]$} \\
miR-31 & RhoA & IL2 induction in T-cells & {$[80]$} \\
miR-3148 & TLR7 & TLR7 inflammatory pathway & {$[63]$} \\
\hline
\end{tabular}

\section{Conclusions}

As we are still at the early stage of the exploration of miRNA's essential roles in SLE pathogenesis, there are many unresolved questions. The application of animal models will advance the field by facilitating the study of the in vivo function of miRNAs in SLE. Profiling the expression of miRNAs still needs to be done to obtain more precise expression patterns of miRNAs among SLE patients with different ages, or different races, or different genders, or different disease manifestations and to identify novel specific biomarkers. The identification of new targets of miRNAs will give us a more comprehensive view of the mechanisms of miRNAs in regulating immune responses in SLE. Developing new techniques to precisely and conveniently measure the expression of miRNAs in our body fluid and novel methods to efficiently overexpress or inhibit the activities of miRNAs in vivo will further accelerate the translational application of miRNAs as novel diagnosis and therapeutic methods in SLE.

\section{Acknowledgments}

Supported by the National Basic Research Program of China (973 program) (2014CB541902), the National Natural Science Foundation of China (No. 81230072; No. 81401331) and the Program of the Shanghai Commission of Science and Technology (No. 12JC1406000).

\section{Conflicts of Interest}

The authors declare no conflict of interest.

\section{References}

1. Rodriguez, A.; Griffiths-Jones, S.; Ashurst, J.L.; Bradley, A. Identification of mammalian microRNA host genes and transcription units. Genome Res. 2004, 14, 1902-1910.

2. Lee, Y.; Jeon, K.; Lee, J.-T.; Kim, S.; Kim, V.N. MicroRNA maturation: Stepwise processing and subcellular localization. EMBO J. 2002, 21, 4663-4670.

3. Kim, V.N.; Han, J.; Siomi, M.C. Biogenesis of small RNAs in animals. Nat. Rev. Mol. Cell Biol. 2009, 10, 126-139.

4. Borchert, G.M.; Lanier, W.; Davidson, B.L. RNA polymerase III transcribes human microRNAs. Nat. Struct. Mol. Biol. 2006, 13, 1097-1101.

5. Lee, Y.; Kim, M.; Han, J.; Yeom, K.H.; Lee, S.; Baek, S.H.; Kim, V.N. MicroRNA genes are transcribed by RNA polymerase II. EMBO J. 2004, 23, 4051-4060.

6. Lee, Y.; Ahn, C.; Han, J.; Choi, H.; Kim, J.; Yim, J.; Lee, J.; Provost, P.; Radmark, O.; Kim, S.; et al. The nuclear rnase III Drosha initiates microRNA processing. Nature 2003, 425, 415-419. 
7. Yi, R.; Qin, Y.; Macara, I.G.; Cullen, B.R. Exportin-5 mediates the nuclear export of pre-microRNAs and short hairpin RNAs. Genes Dev. 2003, 17, 3011-3016.

8. Winter, J.; Jung, S.; Keller, S.; Gregory, R.I.; Diederichs, S. Many roads to maturity: MicroRNA biogenesis pathways and their regulation. Nat. Cell Biol. 2009, 11, 228-234.

9. Khvorova, A.; Reynolds, A.; Jayasena, S.D. Functional siRNAs and miRNAs exhibit strand bias. Cell 2003, 115, 209-216.

10. Yang, J.-S.; Phillips, M.D.; Betel, D.; Mu, P.; Ventura, A.; Siepel, A.C.; Chen, K.C.; Lai, E.C. Widespread regulatory activity of vertebrate microRNA* species. RNA 2011, 17, 312-326.

11. Okamura, K.; Phillips, M.D.; Tyler, D.M.; Duan, H.; Chou, Y.-T.; Lai, E.C. The regulatory activity of microRNA* species has substantial influence on microRNA and 3' UTR evolution. Nat. Struct. Mol. Biol. 2008, 15, 354-363.

12. Kozomara, A.; Griffiths-Jones, S. Mirbase: Annotating high confidence micrornas using deep sequencing data. Nucleic Acids Res. 2014, 42, D68-D73.

13. Krol, J.; Loedige, I.; Filipowicz, W. The widespread regulation of microRNA biogenesis, function and decay. Nat. Rev. Genet. 2010, 11, 597-610.

14. Davis-Dusenbery, B.N.; Hata, A. Mechanisms of control of microRNA biogenesis. J. Biochem. 2010, 148, 381-392.

15. Han, J.; Lee, Y.; Yeom, K.-H.; Nam, J.-W.; Heo, I.; Rhee, J.-K.; Sohn, S.Y.; Cho, Y.; Zhang, B.-T.; Kim, V.N. Molecular basis for the recognition of primary microRNAs by the Drosha-DGCR8 complex. Cell 2006, 125, 887-901.

16. Gregory, R.I.; Yan, K.-P.; Amuthan, G.; Chendrimada, T.; Doratotaj, B.; Cooch, N.; Shiekhattar, R. The microprocessor complex mediates the genesis of microRNAs. Nature 2004, 432, 235-240.

17. Lee, H.Y.; Zhou, K.; Smith, A.M.; Noland, C.L.; Doudna, J.A. Differential roles of human Dicer-binding proteins TRBP and PACT in small RNA processing. Nucleic Acids Res. 2013, 41, 6568-6576.

18. Lee, S.H.; Cho, S.; Sun Kim, M.; Choi, K.; Cho, J.Y.; Gwak, H.S.; Kim, Y.J.; Yoo, H.; Lee, S.H.; Park, J.B.; et al. The ubiquitin ligase human TRIM71 regulates let-7 microRNA biogenesis via modulation of Lin28B protein. Biochim. Biophys. Acta 2014, 1839, 374-386.

19. Heo, I.; Joo, C.; Kim, Y.-K.; Ha, M.; Yoon, M.-J.; Cho, J.; Yeom, K.-H.; Han, J.; Kim, V.N. TUT4 in concert with Lin28 suppresses microRNA biogenesis through pre-microRNA uridylation. Cell 2009, 138, 696-708.

20. Bartel, D.P. MicroRNAs: Target recognition and regulatory functions. Cell 2009, 136, 215-233.

21. Shin, C.; Nam, J.-W.; Farh, K.K.-H.; Chiang, H.R.; Shkumatava, A.; Bartel, D.P. Expanding the microRNA targeting code: Functional sites with centered pairing. Mol. Cell 2010, 38, 789-802.

22. Bhattacharyya, S.N.; Habermacher, R.; Martine, U.; Closs, E.I.; Filipowicz, W. Relief of microRNA-mediated translational repression in human cells subjected to stress. Cell 2006, 125, 1111-1124.

23. Kedde, M.; Strasser, M.J.; Boldajipour, B.; Vrielink, J.A.F.O.; Slanchev, K.; le Sage, C.; Nagel, R.; Voorhoeve, P.M.; van Duijse, J.; Ørom, U.A.; et al. RNA-binding protein DND1 inhibits microRNA access to target mRNA. Cell 2007, 131, 1273-1286. 
24. Memczak, S.; Jens, M.; Elefsinioti, A.; Torti, F.; Krueger, J.; Rybak, A.; Maier, L.; Mackowiak, S.D.; Gregersen, L.H.; Munschauer, M.; et al. Circular RNAs are a large class of animal RNAs with regulatory potency. Nature 2013, 495, 333-338.

25. Rybak, A.; Fuchs, H.; Hadian, K.; Smirnova, L.; Wulczyn, E.A.; Michel, G.; Nitsch, R.; Krappmann, D.; Wulczyn, F.G. The let-7 target gene mouse Lin-41 is a stem cell specific E3 ubiquitin ligase for the miRNA pathway protein Ago2. Nat. Cell Biol. 2009, 11, 1411-1420.

26. Gargalionis, A.N.; Basdra, E.K. Insights in microRNAs biology. Curr. Top. Med. Chem. 2013, 13, 1493-1502.

27. Xiao, C.C.; Rajewsky, K. MicroRNA control in the immune system: Basic principles. Cell 2009, 136, 26-36.

28. Croce, C.M. Causes and consequences of microRNA dysregulation in cancer. Nat. Rev. Genet. 2009, 10, 704-714.

29. Condorelli, G.; Latronico, M.V.; Cavarretta, E. MicroRNAs in cardiovascular diseases: Current knowledge and the road ahead. J. Am. Coll. Cardiol. 2014, 63, 2177-2187.

30. Pauley, K.M.; Cha, S.; Chan, E.K. MicroRNA in autoimmunity and autoimmune diseases. J. Autoimmun. 2009, 32, 189-194.

31. Bhanji, R.A.; Eystathioy, T.; Chan, E.K.; Bloch, D.B.; Fritzler, M.J. Clinical and serological features of patients with autoantibodies to GW/P bodies. Clin. Immunol. 2007, 125, 247-256.

32. Jakymiw, A.; Ikeda, K.; Fritzler, M.J.; Reeves, W.H.; Satoh, M.; Chan, E.K. Autoimmune targeting of key components of RNA interference. Arthritis Res. Ther. 2006, 8, doi:10.1186/ar1959.

33. Dai, Y.; Huang, Y.S.; Tang, M.; Lv, T.Y.; Hu, C.X.; Tan, Y.H.; Xu, Z.M.; Yin, Y.B. Microarray analysis of microRNA expression in peripheral blood cells of systemic lupus erythematosus patients. Lupus 2007, 16, 939-946.

34. Dai, R.; Zhang, Y.; Khan, D.; Heid, B.; Caudell, D.; Crasta, O.; Ahmed, S.A. Identification of a common lupus disease-associated microRNA expression pattern in three different murine models of lupus. PLoS ONE 2010, 5, e14302.

35. Akira, S.; Uematsu, S.; Takeuchi, O. Pathogen recognition and innate immunity. Cell 2006, 124, 783-801.

36. Lamkanfi, M.; Dixit, V.M. Inflammasomes and their roles in health and disease. Ann. Rev. Cell Dev. Biol. 2012, 28, 137-161.

37. Kawai, T.; Akira, S. The role of pattern-recognition receptors in innate immunity: Update on toll-like receptors. Nat. Immunol. 2010, 11, 373-384.

38. Barber, G.N. Innate immune DNA sensing pathways: Sting, aimii and the regulation of interferon production and inflammatory responses. Curr. Opin. Immunol. 2011, 23, 10-20.

39. Medzhitov, R.; Janeway, C.A., Jr. Innate immunity: Impact on the adaptive immune response. Curr. Opin. Immunol. 1997, 9, 4-9.

40. Rifkin, I.R.; Leadbetter, E.A.; Busconi, L.; Viglianti, G.; Marshak-Rothstein, A. Toll-like receptors, endogenous ligands, and systemic autoimmune disease. Immunol. Rev. 2005, 204, $27-42$.

41. Leadbetter, E.A.; Rifkin, I.R.; Hohlbaum, A.M.; Beaudette, B.C.; Shlomchik, M.J.; Marshak-Rothstein, A. Chromatin-IgG complexes activate B cells by dual engagement of IgM and toll-like receptors. Nature 2002, 416, 603-607. 
42. Anders, H.-J.; Vielhauer, V.; Eis, V.; Linde, Y.; Kretzler, M.; Perez de Lema, G.; Strutz, F.; Bauer, S.; Rutz, M.; Wagner, H.; et al. Activation of toll-like receptor-9 induces progression of renal disease in MRL-Fas(lpr) mice. FASEB J. 2004, 19, 534-536.

43. Banchereau, J.; Pascual, V. Type I interferon in systemic lupus erythematosus and other autoimmune diseases. Immunity 2006, 25, 383-392.

44. Baccala, R.; Hoebe, K.; Kono, D.H.; Beutler, B.; Theofilopoulos, A.N. TLR-dependent and TLR-independent pathways of type i interferon induction in systemic autoimmunity. Nat. Med. 2007, 13, 543-551.

45. Shrivastav, M.; Niewold, T.B. Nucleic acid sensors and type I interferon production in systemic lupus erythematosus. Front. Immunol. 2013, 4, doi:10.3389/fimmu.2013.00319.

46. Lee, P.Y.; Kumagai, Y.; Li, Y.; Takeuchi, O.; Yoshida, H.; Weinstein, J.; Kellner, E.S.; Nacionales, D.; Barker, T.; Kelly-Scumpia, K. TLR7-dependent and FCgammaR-independent production of type I interferon in experimental mouse lupus. J. Exp. Med. 2008, 205, 2995-3006.

47. Subramanian, S.; Tus, K.; Li, Q.-Z.; Wang, A.; Tian, X.-H.; Zhou, J.; Liang, C.; Bartov, G.; McDaniel, L.D.; Zhou, X.J.; et al. A TLR7 translocation accelerates systemic autoimmunity in murine lupus. Proc. Natl. Acad. Sci. USA 2006, 103, 9970-9975.

48. Han, G.; Chen, S.; Shen, N.; Ye, S.; Bao, C.; Gu, Y. Analysis of gene expression profiles in human systemic lupus erythematosus using oligonucleotide microarray. Genes Immun. 2003, 4, 177-186.

49. Barber, G.N. Sting-dependent cytosolic DNA sensing pathways. Trends Immunol. 2014, 35, 88-93.

50. Crow, Y.J.; Hayward, B.E.; Parmar, R.; Robins, P.; Leitch, A.; Ali, M.; Black, D.N.; van Bokhoven, H.; Brunner, H.G.; Hamel, B.C.; et al. Mutations in the gene encoding the 3'-5' DNA exonuclease TREX1 cause aicardi-goutieres syndrome at the AGS1 locus. Nat. Genet. 2006, 38, 917-920.

51. Stetson, D.B.; Ko, J.S.; Heidmann, T.; Medzhitov, R. Trex1 prevents cell-intrinsic initiation of autoimmunity. Cell 2008, 134, 587-598.

52. Gall, A.; Treuting, P.; Elkon, K.B.; Loo, Y.-M.; Gale Jr, M.; Barber, G.N.; Stetson, D.B. Autoimmunity initiates in non-hematopoietic cells and progresses via lymphocytes in an interferon-dependent autoimmune disease. Immunity 2012, 36, 120.

53. Taganov, K.D.; Boldin, M.P.; Chang, K.-J.; Baltimore, D. NF- $\kappa$ B-dependent induction of microRNA miR-146, an inhibitor targeted to signaling proteins of innate immune responses. Proc. Natl. Acad. Sci. USA 2006, 103, 12481-12486.

54. Hou, J.; Wang, P.; Lin, L.; Liu, X.; Ma, F.; An, H.; Wang, Z.; Cao, X. MicroRNA-146a feedback inhibits RIG-I-dependent type I IFN production in macrophages by targeting TRAF6, IRAK1, and IRAK2. J. Immunol. 2009, 183, 2150-2158.

55. Tang, Y.; Luo, X.; Cui, H.; Ni, X.; Yuan, M.; Guo, Y.; Huang, X.; Zhou, H.; de Vries, N.; Tak, P.P. MicroRNA-146a contributes to abnormal activation of the type I interferon pathway in human lupus by targeting the key signaling proteins. Arthritis Rheumatol. 2009, 60, 1065-1075.

56. Luo, X.; Yang, W.; Ye, D.-Q.; Cui, H.; Zhang, Y.; Hirankarn, N.; Qian, X.; Tang, Y.; Lau, Y.L.; de Vries, N.; et al. A functional variant in microRNA-146a promoter modulates its expression and confers disease risk for systemic lupus erythematosus. PLoS Genet. 2011, 7, e1002128. 
57. Tang, B.; Xiao, B.; Liu, Z.; Li, N.; Zhu, E.D.; Li, B.S.; Xie, Q.H.; Zhuang, Y.; Zou, Q.M.; Mao, X.H. Identification of MyD88 as a novel target of miR-155, involved in negative regulation of Helicobacter pylori-induced inflammation. FEBS Lett. 2010, 584, 1481-1486.

58. Ceppi, M.; Pereira, P.M.; Dunand-Sauthier, I.; Barras, E.; Reith, W.; Santos, M.A.; Pierre, P. MicroRNA-155 modulates the interleukin-1 signaling pathway in activated human monocyte-derived dendritic cells. Proc. Natl. Acad. Sci. USA 2009, 106, 2735-2740.

59. Wang, P.; Hou, J.; Lin, L.; Wang, C.; Liu, X.; Li, D.; Ma, F.; Wang, Z.; Cao, X. Inducible microRNA-155 feedback promotes type I IFN signaling in antiviral innate immunity by targeting suppressor of cytokine signaling 1. J. Immunol. 2010, 185, 6226-6233.

60. Zhou, H.; Huang, X.; Cui, H.; Luo, X.; Tang, Y.; Chen, S.; Wu, L.; Shen, N. miR-155 and its star-form partner miR-155* cooperatively regulate type I interferon production by human plasmacytoid dendritic cells. Blood 2010, 116, 5885-5894.

61. Gilliet, M.; Cao, W.; Liu, Y.-J. Plasmacytoid dendritic cells: Sensing nucleic acids in viral infection and autoimmune diseases. Nat. Rev. Immunol. 2008, 8, 594-606.

62. Rowland, S.L.; Riggs, J.M.; Gilfillan, S.; Bugatti, M.; Vermi, W.; Kolbeck, R.; Unanue, E.R.; Sanjuan, M.A.; Colonna, M. Early, transient depletion of plasmacytoid dendritic cells ameliorates autoimmunity in a lupus model. J. Exp. Med. 2014, 211, 1977-1991.

63. Deng, Y.; Zhao, J.; Sakurai, D.; Kaufman, K.M.; Edberg, J.C.; Kimberly, R.P.; Kamen, D.L.; Gilkeson, G.S.; Jacob, C.O.; Scofield, R.H.; et al. MicroRNA-3148 modulates allelic expression of toll-like receptor 7 variant associated with systemic lupus erythematosus. PLoS Genet. 2013, 9, e1003336.

64. Kim, S.J.; Gregersen, P.K.; Diamond, B. Regulation of dendritic cell activation by microRNA let-7c and blimp1. J. Clin. Investig. 2013, 123, 823-833.

65. Chafin, C.B.; Regna, N.L.; Dai, R.; Caudell, D.L.; Reilly, C.M. MicroRNA-let-7a expression is increased in the mesangial cells of NZB/W mice and increases IL-6 production in vitro. Autoimmunity 2013, 46, 351-362.

66. Crispin, J.C.; Kyttaris, V.C.; Terhorst, C.; Tsokos, G.C. T cells as therapeutic targets in SLE. Nat. Rev. Rheumatol. 2010, 6, 317-325.

67. Coca, A.; Sanz, I. Updates on B-cell immunotherapies for systemic lupus erythematosus and sjogren's syndrome. Curr. Opin. Rheumatol. 2012, 24, 451-456.

68. Pugh-Bernard, A.E.; Cambier, J.C. B cell receptor signaling in human systemic lupus erythematosus. Curr. Opin. Rheumatol. 2006, 18, 451-455.

69. Liu, Z.; Davidson, A. Baff and selection of autoreactive B cells. Trends Immunol. 2011, 32, 388-394.

70. Richardson, B.; Scheinbart, L.; Strahler, J.; Gross, L.; Hanash, S.; Johnson, M. Evidence for impaired T cell DNA methylation in systemic lupus erythematosus and rheumatoid arthritis. Arthritis Rheumatol. 1990, 33, 1665-1673.

71. Pan, W.; Zhu, S.; Yuan, M.; Cui, H.; Wang, L.; Luo, X.; Li, J.; Zhou, H.; Tang, Y.; Shen, N. MicroRNA-21 and microRNA-148a contribute to DNA hypomethylation in lupus CD4+ T cells by directly and indirectly targeting DNA methyltransferase 1. J. Immunol. 2010, 184, 6773-6781.

72. Ballestar, E.; Esteller, M.; Richardson, B.C. The epigenetic face of systemic lupus erythematosus. J. Immunol. 2006, 176, 7143-7147. 
73. Zhao, S.; Wang, Y.; Liang, Y.; Zhao, M.; Long, H.; Ding, S.; Yin, H.; Lu, Q. MicroRNA-126 regulates DNA methylation in CD4+ T cells and contributes to systemic lupus erythematosus by targeting DNA methyltransferase 1. Arthritis Rheumatol. 2011, 63, 1376-1386.

74. Qin, H.; Zhu, X.; Liang, J.; Wu, J.; Yang, Y.; Wang, S.; Shi, W.; Xu, J. MicroRNA-29b contributes to DNA hypomethylation of CD4+ T cells in systemic lupus erythematosus by indirectly targeting DNA methyltransferase 1. J. Dermatol. Sci. 2013, 69, 61-67.

75. Alcocer-Varela, J.; Alarcon-Segovia, D. Decreased production of and response to interleukin-2 by cultured lymphocytes from patients with systemic lupus erythematosus. J. Clin. Investig. 1982, 69, 1388.

76. Setoguchi, R.; Hori, S.; Takahashi, T.; Sakaguchi, S. Homeostatic maintenance of natural Foxp ${ }^{3+}$ $\mathrm{CD} 25^{+} \mathrm{CD}^{+}$regulatory $\mathrm{T}$ cells by interleukin (IL) -2 and induction of autoimmune disease by il-2 neutralization. J. Exp. Med. 2005, 201, 723-735.

77. Lu, M.M.; Wang, J.; Pan, H.F.; Chen, G.M.; Li, J.; Cen, H.; Feng, C.C.; Ye, D.Q. Increased serum RANTES in patients with systemic lupus erythematosus. Rheumatol. Int. 2012, 32, 1231-1233.

78. De Lema, G.P.; Maier, H.; Nieto, E.; Vielhauer, V.; Luckow, B.; Mampaso, F.; Schlöndorff, D. Chemokine expression precedes inflammatory cell infiltration and chemokine receptor and cytokine expression during the initiation of murine lupus nephritis. J. Am. Soc. Nephrol. 2001, 12, 1369-1382.

79. Crispin, J.C.; Tsokos, G.C. Transcriptional regulation of IL-2 in health and autoimmunity. Autoimmun. Rev. 2009, 8, 190-195.

80. Fan, W.; Liang, D.; Tang, Y.; Qu, B.; Cui, H.; Luo, X.; Huang, X.; Chen, S.; Higgs, B.W.; Jallal, B. Identification of microRNA-31 as a novel regulator contributing to impaired interleukin-2 production in t cells from patients with systemic lupus erythematosus. Arthritis Rheumatol. 2012, $64,3715-3725$.

81. Zhao, X.; Tang, Y.; Qu, B.; Cui, H.; Wang, S.; Wang, L.; Luo, X.; Huang, X.; Li, J.; Chen, S. MicroRNA-125A contributes to elevated inflammatory chemokine rantes levels via targeting KLF13 in systemic lupus erythematosus. Arthritis Rheumatol. 2010, 62, 3425-3435.

82. Grammer, A.C.; Slota, R.; Fischer, R.; Gur, H.; Girschick, H.; Yarboro, C.; Illei, G.G.; Lipsky, P.E. Abnormal germinal center reactions in systemic lupus erythematosus demonstrated by blockade of CD154-CD40 interactions. J. Clin. Investig. 2003, 112, 1506-1520.

83. Crotty, S. Follicular helper CD4 T cells (TFH). Annu. Rev. Immunol. 2011, 29, 621-663.

84. Xiao, C.; Srinivasan, L.; Calado, D.P.; Patterson, H.C.; Zhang, B.; Wang, J.; Henderson, J.M.; Kutok, J.L.; Rajewsky, K. Lymphoproliferative disease and autoimmunity in mice with increased miR-17-92 expression in lymphocytes. Nat. Immunol. 2008, 9, 405-414.

85. Baumjohann, D.; Kageyama, R.; Clingan, J.M.; Morar, M.M.; Patel, S.; de Kouchkovsky, D.; Bannard, O.; Bluestone, J.A.; Matloubian, M.; Ansel, K.M.; et al. The microRNA cluster miR-17(sim)92 promotes tfh cell differentiation and represses subset-inappropriate gene expression. Nat. Immunol. 2013, 14, 840-848.

86. Kang, S.G.; Liu, W.-H.; Lu, P.; Jin, H.Y.; Lim, H.W.; Shepherd, J.; Fremgen, D.; Verdin, E.; Oldstone, M.B.A.; Qi, H.; et al. MicroRNAs of the miR-17(SIM)92 family are critical regulators of TFH differentiation. Nat. Immunol. 2013, 14, 849-857. 
87. Liu, Y.; Dong, J.; Mu, R.; Gao, Y.; Tan, X.; Li, Y.; Li, Z.; Yang, G. MicroRNA-30Apromotes B cell hyperactivity in patients with systemic lupus erythematosus by direct interaction with Lyn. Arthritis Rheumatol. 2013, 65, 1603-1611.

88. Kotake, S.; Udagawa, N.; Takahashi, N.; Matsuzaki, K.; Itoh, K.; Ishiyama, S.; Saito, S.; Inoue, K.; Kamatani, N.; Gillespie, M.T. IL-17 in synovial fluids from patients with rheumatoid arthritis is a potent stimulator of osteoclastogenesis. J. Clin. Investig. 1999, 103, 1345-1352.

89. Komiyama, Y.; Nakae, S.; Matsuki, T.; Nambu, A.; Ishigame, H.; Kakuta, S.; Sudo, K.; Iwakura, Y. IL-17 plays an important role in the development of experimental autoimmune encephalomyelitis. J. Immunol. 2006, 177, 566-573.

90. Wong, C.; Ho, C.Y.; Li, E.; Lam, C. Elevation of proinflammatory cytokine (IL-18, IL-17, IL-12) and th2 cytokine (IL-4) concentrations in patients with systemic lupus erythematosus. Lupus 2000, 9, 589-593.

91. Zhu, S.; Pan, W.; Song, X.; Liu, Y.; Shao, X.; Tang, Y.; Liang, D.; He, D.; Wang, H.; Liu, W.; et al. The microRNA miR-23b suppresses IL-17-associated autoimmune inflammation by targeting TAB2, TAB3 and IKK- $\alpha$. Nat. Med. 2012, 18, 1077-1086.

92. Cameron, J.S. Lupus nephritis. J. Am. Soc. Nephrol. 1999, 10, 413-424.

93. Dai, Y.; Sui, W.; Lan, H.; Yan, Q.; Huang, H.; Huang, Y. Comprehensive analysis of microRNA expression patterns in renal biopsies of lupus nephritis patients. Rheumatol. Int. 2009, 29, 749-754.

94. Zhou, H.; Hasni, S.A.; Perez, P.; Tandon, M.; Jang, S.I.; Zheng, C.; Kopp, J.B.; Austin, H., 3rd; Balow, J.E.; Alevizos, I.; et al. MiR-150 promotes renal fibrosis in lupus nephritis by downregulating socs1. J. Am. Soc. Nephrol. JASN 2013, 24, 1073-1087.

95. Liu, C.-C.; Kao, A.H.; Manzi, S.; Ahearn, J.M. Biomarkers in systemic lupus erythematosus: Challenges and prospects for the future. Ther. Adv. Musculoskelet. Dis. 2013, 5, 210-233.

96. Stagakis, E.; Bertsias, G.; Verginis, P.; Nakou, M.; Hatziapostolou, M.; Kritikos, H.; Iliopoulos, D.; Boumpas, D.T. Identification of novel microrna signatures linked to human lupus disease activity and pathogenesis: MiR-21 regulates aberrant $t$ cell responses through regulation of pdcd4 expression. Ann. Rheum. Dis. 2011, 70, 1496-1506.

97. Sui, W.; Liu, F.; Chen, J.; Ou, M.; Dai, Y. Microarray technology for analysis of microrna expression in renal biopsies of lupus nephritis patients. Methods Mol. Biol. 2014, 1134, 211-220.

98. Chauhan, S.K.; Singh, V.V.; Rai, R.; Rai, M.; Rai, G. Differential microrna profile and post-transcriptional regulation exist in systemic lupus erythematosus patients with distinct autoantibody specificities. J. Clin. Immunol. 2014, 34, 491-503.

99. Te, J.L.; Dozmorov, I.M.; Guthridge, J.M.; Nguyen, K.L.; Cavett, J.W.; Kelly, J.A.; Bruner, G.R.; Harley, J.B.; Ojwang, J.O. Identification of unique microRNA signature associated with lupus nephritis. PLOS ONE 2010, 5, e10344.

100. Kosaka, N.; Iguchi, H.; Ochiya, T. Circulating microRNA in body fluid: A new potential biomarker for cancer diagnosis and prognosis. Cancer Sci. 2010, 101, 2087-2092.

101. Wang, G.-K.; Zhu, J.-Q.; Zhang, J.-T.; Li, Q.; Li, Y.; He, J.; Qin, Y.-W.; Jing, Q. Circulating microRNA: A novel potential biomarker for early diagnosis of acute myocardial infarction in humans. Eur. Heart J. 2010, 31, 659-666.

102. Wang, G.; Tam, L.; Li, E.; Kwan, B.; Chow, K.; Luk, C.; Li, P.; Szeto, C. Serum and urinary free microrna level in patients with systemic lupus erythematosus. Lupus 2011, 20, 493-500. 
103. Carlsen, A.L.; Schetter, A.J.; Nielsen, C.T.; Lood, C.; Knudsen, S.; Voss, A.; Harris, C.C.; Hellmark, T.; Segelmark, M.; Jacobsen, S.; et al. Circulating microRNA expression profiles associated with systemic lupus erythematosus. Arthritis Rheumatol. 2013, 65, 1324-1334.

104. Chen, W.; Tan, K.; Huang, J.; Yu, X.; Peng, W.; Chen, Y.; Lin, X.; Chen, D.; Dai, Y. Analysis of microRNAs in patients with systemic lupus erythematosus, using solexa deep sequencing. Connect. Tissue Res. 2014, 55, 187-196.

105. Tang, Q.; Yang, Y.; Zhao, M.; Liang, G.; Wu, H.; Liu, Q.; Xie, Y.; Li, D.; Dai, Y.; Yung, S.; et al. Mycophenolic acid upregulates miR-142-3P/5P and miR-146a in lupus CD4+ T cells. Lupus 2015, doi:10.1177/0961203315570685

106. Thai, T.-H.; Calado, D.P.; Casola, S.; Ansel, K.M.; Xiao, C.; Xue, Y.; Murphy, A.; Frendewey, D.; Valenzuela, D.; Kutok, J.L.; et al. Regulation of the germinal center response by microRNA-155. Science 2007, 316, 604-608.

107. Vigorito, E.; Perks, K.L.; Abreu-Goodger, C.; Bunting, S.; Xiang, Z.; Kohlhaas, S.; Das, P.P.; Miska, E.A.; Rodriguez, A.; Bradley, A.; et al. MicroRNA-155 regulates the generation of immunoglobulin class-switched plasma cells. Immunity 2007, 27, 847-859.

108. Thai, T.H.; Patterson, H.C.; Pham, D.H.; Kis-Toth, K.; Kaminski, D.A.; Tsokos, G.C. Deletion of microRNA-155 reduces autoantibody responses and alleviates lupus-like disease in the fas(lpr) mouse. Proc. Natl. Acad. Sci. USA 2013, 110, 20194-20199.

109. Zhou, S.Y.; Liang, D.; Huang, X.F.; Xiao, C.Y.; Tang, Y.J.; Jia, Q.; Harley, J.B.; Shen, N. In vivo therapeutic success of microRNA-155 (miR-155) antagomir in a mouse model of lupus pulmonary hemorrhage. Arthritis Rheumatol. 2013, 65, S246-S247.

110. Lashine, Y.A.; Salah, S.; Aboelenein, H.R.; Abdelaziz, A.I. Correcting the expression of miRNA-155 represses PP2AC and enhances the release of IL-2 in pbmcs of juvenile SLE patients. Lupus 2015, 24, 240-247.

111. Garchow, B.G.; Bartulos Encinas, O.; Leung, Y.T.; Tsao, P.Y.; Eisenberg, R.A.; Caricchio, R.; Obad, S.; Petri, A.; Kauppinen, S.; Kiriakidou, M. Silencing of microRNA-21 in vivo ameliorates autoimmune splenomegaly in lupus mice. EMBO Mol. Med. 2011, 3, 605-615.

112. Lu, L.-F.; Boldin, M.P.; Chaudhry, A.; Lin, L.-L.; Taganov, K.D.; Hanada, T.; Yoshimura, A.; Baltimore, D.; Rudensky, A.Y. Function of miR-146a in controlling TREG cell-mediated regulation of TH1 responses. Cell 2010, 142, 914-929.

113. Boldin, M.P.; Taganov, K.D.; Rao, D.S.; Yang, L.; Zhao, J.L.; Kalwani, M.; Garcia-Flores, Y.; Luong, M.; Devrekanli, A.; Xu, J. MiR-146a is a significant brake on autoimmunity, myeloproliferation, and cancer in mice. J. Exp. Med. 2011, 208, 1189-1201.

114. Liang, D.; Zhou, S.Y.; Liu, Z.; Shan, Z.Y.; Brohawn, P.; Yao, Y.H.; Harley, J.B.; Shen, N. In vivo administration of miR-146a protects $\mathrm{C} 57 \mathrm{BL} / 6$ mice from pristane-induced pulmonary hemorrhage via suppressing type i interferon response. Arthritis Rheumatol. 2013, 65, S1162-S1162.

115. Van Rooij, E.; Purcell, A.L.; Levin, A.A. Developing microRNA therapeutics. Circ. Res. 2012, 110, 496-507.

(C) 2015 by the authors; licensee MDPI, Basel, Switzerland. This article is an open access article distributed under the terms and conditions of the Creative Commons Attribution license (http://creativecommons.org/licenses/by/4.0/). 